Secretome of Mesenchymal Progenitors from the Umbilical Cord Acts as Modulator of Neural/Glial Proliferation and Differentiation

Fábio G. Teixeira, Miguel M. Carvalho, Andreia Neves-Carvalho, Krishna M. Panchalingam, Leo A. Behie, Luísa Pinto, Nuno Sousa, et al.

Stem Cell Reviews and Reports

ISSN 1550-8943

Volume 11

Number 2

Stem Cell Rev and Rep (2015)

11:288-297

DOI 10.1007/s12015-014-9576-2

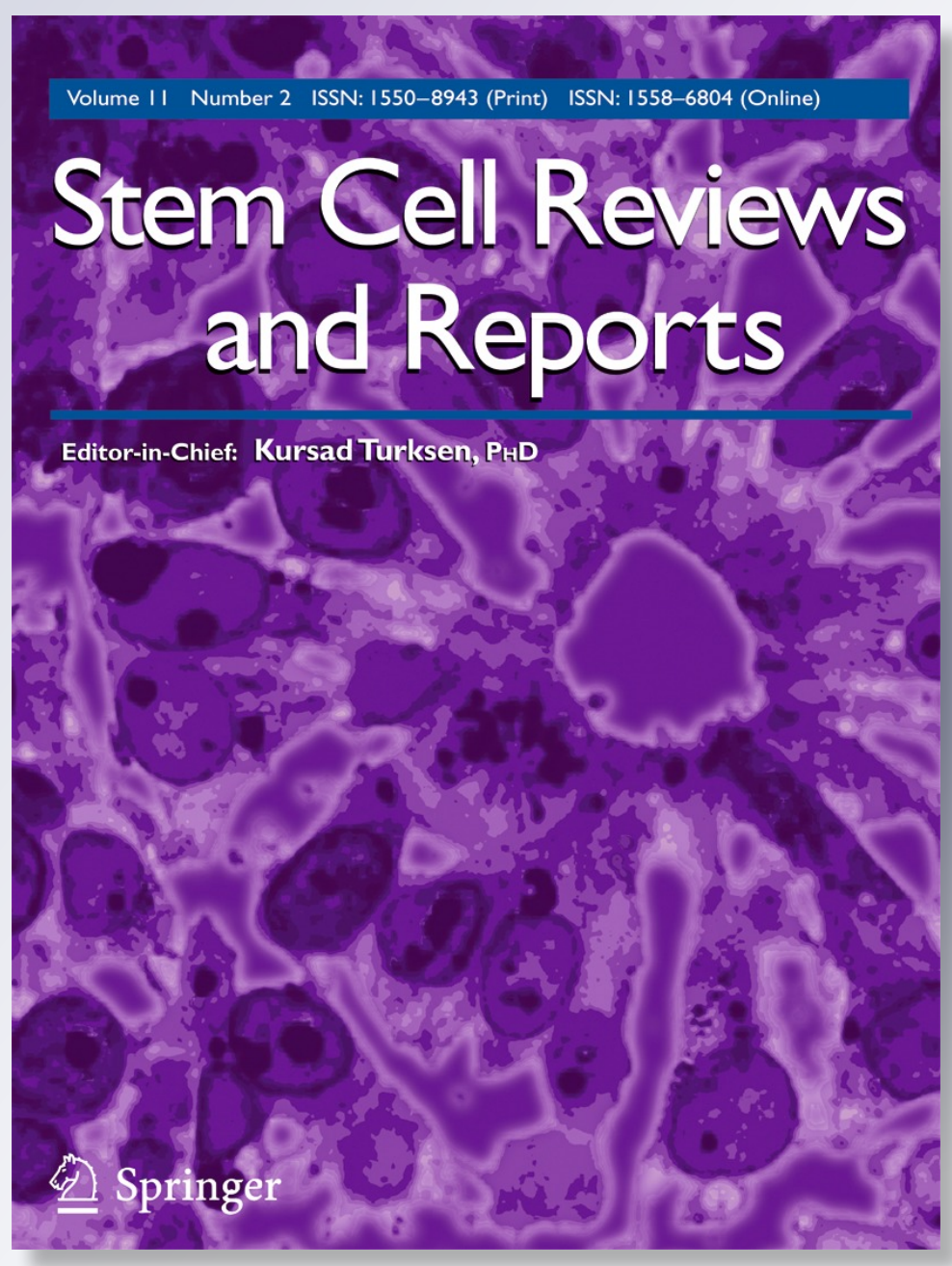

望 Springer 
Your article is protected by copyright and all rights are held exclusively by Springer Science +Business Media New York. This e-offprint is for personal use only and shall not be selfarchived in electronic repositories. If you wish to self-archive your article, please use the accepted manuscript version for posting on your own website. You may further deposit the accepted manuscript version in any repository, provided it is only made publicly available 12 months after official publication or later and provided acknowledgement is given to the original source of publication and a link is inserted to the published article on Springer's website. The link must be accompanied by the following text: "The final publication is available at link.springer.com". 


\title{
Secretome of Mesenchymal Progenitors from the Umbilical Cord Acts as Modulator of Neural/Glial Proliferation and Differentiation
}

\author{
Fábio G. Teixeira • Miguel M. Carvalho • Andreia Neves-Carvalho • \\ Krishna M. Panchalingam • Leo A. Behie - Luísa Pinto • \\ Nuno Sousa • António J. Salgado
}

Published online: 25 November 2014

(C) Springer Science+Business Media New York 2014

\begin{abstract}
It was recently shown that the conditioned media (CM) of Human Umbilical Cord Perivascular Cells (HUCPVCs), a mesenchymal progenitor population residing within the Wharton Jelly of the umbilical cord, was able to modulate in vitro the survival and viability of different neuronal and glial cells populations. In the present work, we aimed to assess if the secretome of HUCPVCs is able to 1) induce the differentiation of human telencephalon neural precursor cells (htNPCs) in vitro, and 2) modulate neural/glial proliferation, differentiation and survival in the dentate gyrus (DG) of adult rat hippocampus. For this purpose, two separate experimental setups were performed: 1) htNPCs were incubated with HUCPVCs-CM for 5 days after which neuronal differentiation was assessed and, 2) HUCPVCs, or their respective CM, were injected into the DG of young adult rats and their effects assessed 7 days later. Results revealed that the secretome of HUCPVCs was able to increase neuronal cell differentiation in vitro; indeed, higher densities of immature $\left(\mathrm{DCX}^{+}\right.$cells) and mature neurons (MAP- $2^{+}$cells) were observed when htNPCs were incubated with the HUCPVCs-CM. Additionally, when HUCPVCs and their CM were injected in the DG, results revealed that both cells or CM were able to increase the endogenous proliferation $\left(\mathrm{BrdU}^{+}\right.$cells) 7 days
\end{abstract}

F. G. Teixeira $\cdot$ M. M. Carvalho $\cdot$ A. Neves-Carvalho $\cdot$ L. Pinto $•$

N. Sousa $\cdot$ A. J. Salgado $(\bowtie)$

Life and Health Sciences Research Institute (ICVS), School of Health

Sciences, University of Minho, Braga, Portugal

e-mail: asalgado@ecsaude.uminho.pt

F. G. Teixeira • M. M. Carvalho - A. Neves-Carvalho $\cdot$ L. Pinto •

N. Sousa $\cdot$ A. J. Salgado

ICVS/3B's - PT Government Associate Laboratory,

Braga/Guimarães, Portugal

K. M. Panchalingam $\cdot$ L. A. Behie

Pharmaceutical Production Research Facility (PPRF), University of

Calgary, Calgary, Canada after injection. It was also possible to observe an increased number of newborn neurons ( $\mathrm{DCX}^{+}$cells), upon injection of HUCPVCs or their respective CM. Finally western blot analysis revealed that after CM or HUCPVCs transplantation, there was an increase of fibroblast growth factor-2 (FGF-2) and, to a lesser extent, of nerve growth factor (NGF) in the DG tissue. Concluding, our results have shown that the transplantation of HUCPVCs or the administration of their secretome were able to potentiate neuronal survival and differentiation in vitro and in vivo.

Keywords CNS $\cdot$ Neurogenesis $\cdot$ MSCs $\cdot$ HUCPVCs $\cdot$ Secretome

\section{Introduction}

It is well established that the mammalian adult brain has the ability to generate new neurons [1]. In adult mammals, the active neurogenic process occurs mainly in the subependymal zone (SEZ) of the lateral ventricles and in the subgranular zone (SGZ) of the hippocampal dentate gyrus (DG) [2, 3]. Nevertheless, the fact that adult neurogenesis is restricted to specific brain regions implies a low regeneration capacity of the central nervous system (CNS) [4]. Therefore, there has been an increasing interest on the development of therapies to promote CNS regeneration. Several studies have proposed the use of adult stem cells as a possible tool for CNS regeneration, and within it, mesenchymal stem cells (MSCs) [4-6] have emerged as a promising therapeutic option [7-9]. In fact, over the last decade, there has been a substantial effort to assess the impact of MSCs for CNS repair [10-12]. Different studies have demonstrated that bone-marrow (BM-MSCs) and adipose stem cells (ASCs) produce a significant recovery of neurological impairments in animal models of stroke [13, 
14], demyelination [15, 16], Parkinson's disease (PD) [17, 18] and spinal cord injury (SCI) $[19,20]$.

The stem/progenitors cells present in the Wharton Jelly of the umbilical cord, known as Wharton Jelly Stem Cells (WJMSCs) and Human Umbilical Cord Perivascular Cells (HUCPVCs), have been suggested as possible populations of interest for CNS applications [21-23]. Like BM-MSCs and ASCs, these populations are also defined as MSCs [24-26]. They are plastic adherent, positive for MSCs' markers (CD73, CD90 and CD105), negative for hematopoietic markers (CD45, CD34, CD14) and they also have the capacity to differentiate towards osteogenic, chondrogenic, and adipogenic lineages [25-27]. Concerning its application into the CNS, Weiss and coworkers [28] revealed that MSCs isolated from the bulk of the porcine WJ matrix, were able to survive in the brains of rats after transplantation without concomitant immunosuppressive therapy. In addition, it was also shown that these stem cells were able to improve the condition of hemiparkinsonian rats [28], to modulate the inflammatory brain reaction after global ischemia [29] as well as, to potentiate axonal regeneration on spinal cord injury $(\mathrm{SCI})[30,31]$. However, little is known regarding the real mechanisms underlying the interactions between umbilical cord stem cell populations and the residing neuronal and glial cells. Recently, it has been suggested that most of the effects that may be promoted by these cells could be mediated by their secretome [23, 32-34]. For instance, in a stroke model, Koh et al. [35] and Ding et al. [36] showed that, after transplantation, MSCs were able to express granulocyte colonystimulating factor (G-CSF), vascular endothelial growth factor (VEGF), glial-derived neurotrophic factor (GDNF), stem cellderived factor 1 (SDF-1) and brain-derived neurotrophic factor (BNDF). Moreover, the authors considered that these growth factors were the main mediatiors for the neuroprotection and reduction of the lesion site as well as, for the improvement of animal behavior and neuroplasticity [35]. Similar findings were also reported in animal models of PD, demonstrating that after cell transplantation there was an increase on the local tissue expression of GDNF and fibroblast growth factor 20 (FGF20 ), leading to the protection of tyrosine hydroxylase (TH) cells and to behavioral amelioration [28]. Adding to this, we have recently shown that the secretome of these cells was able to increase, in vitro, the survival of neuronal and glial populations through the secretion of different trophic factors and, possibly, extracellular vesicles $[33,37]$. Thus, based on these results, we wanted to further explore if the secretome of mesenchymal progenitors isolated from the Wharton Jelly (HUCPVCs), would be able to: 1) induce neuronal differentiation of human telencephalon neural progenitors (htNPCs) and 2) modulate phenomena such as neuronal/glial survival and differentiation in the hippocampal neurogenic niche (DG), upon an injection in the form of conditioned media $(\mathrm{CM})$ or after HUCPVCs transplantation.
Results revealed that the secretome of HUCPVCs was able to induce neuronal differentiation of htNPCs in vitro. Moreover, animals that were injected with the secretome of the cells under study into the hippocampal DG, displayed similar levels of proliferation, neuronal/glial survival and differentiation comparing to those transplanted with HUCPVCs. Finally the injection of the secretome also lead to an enhanced expression of FGF-2 in the resident DG.

\section{Materials and Methods}

\section{Cell Culture}

\section{Isolation and Primary Culture of Human Umbilical Cord Perivascular Stem Cells (HUCPVCs)}

HUCPVCs were obtained from Prof. John E. Davies (University of Toronto, Toronto Canada). Cells were isolated from umbilical cords collected after c-section, with the consent of parenting entities. The isolation of HUCPVCs was performed based on the protocol established by Sarugaser et al. [27]. Briefly, the umbilical cord was cut into small segments $(4-5 \mathrm{~cm})$ to expose both arteries and vein, rejecting the epithelium. Once isolated the vessels, with a sterilized suture, the ends of the vessels were fixed creating a short loop in order to reduce the contamination by erythrocytes. Vessels were then placed in a collagenase solution $(0.5 \mathrm{mg} / \mathrm{ml}$ for arteries and $0.75 \mathrm{mg} / \mathrm{ml}$ for veins; Sigma, USA) and incubated at $37^{\circ} \mathrm{C}$ for a period of $16-18 \mathrm{~h}$. Completed this period, the vessels were removed from the suspension and diluted in PBS without $\mathrm{Mg}^{2+} / \mathrm{Ca}^{2+}$ (Invitrogen, USA). Finally, HUCPVCs were ressuspended in Alpha-Mem medium (Invitrogen, USA) supplemented with $1 \%$ of antibiotic/ antimycotic (Invitrogen, USA) and $10 \%$ of fetal bovine serum (FBS) (Invitrogen, USA), and plated on culture at a density of $4.0 \times 10^{3}$ cells $/ \mathrm{cm}^{2}$. Subsequently, the culture medium was renewed every 3 days and the culture maintained at $37^{\circ} \mathrm{C}, 5 \%$ $\mathrm{CO}_{2}, 95 \%$ air and $90 \%$ relative humidity until confluence.

Mesenchymal phenotype of HUCPVCs was screened by differentiating them towards osteogenic and adipogenic lineages. Cells were incubated with Mesencult ${ }^{\circledR}$ MSC Basal medium supplemented with Mesencult ${ }^{\circledR}$ adipogenic stimulatory supplements or Mesencult ${ }^{\circledR}$ osteogenic kit (Stem Cell Technologies, Canada) during 2 weeks. At the end of this period, osteogenic differentiation was assessed through Alizarin Red staining (Sigma, USA), while adipogenic differentiation was assessed through Oil Red O staining (Sigma, USA).

\section{HUCPVCs Conditioned Media (HUCPVCs-CM) Collection}

The conditioned media (CM) used was collected from HUCPVCs cultures in passage five (P5) previously plated and kept at a density of $4.0 \times 10^{3}$ cells $/ \mathrm{cm}^{2}$ for 3 days in 
Alpha-Mem medium (Invitrogen, USA) supplemented with $1 \%$ of antibiotic/antimycotic (Invitrogen, USA) and $10 \%$ FBS (Invitrogen, USA). After this, the flasks were washed three times in Neurobasal A medium (Invitrogen, USA) for 5 min and then, washed five times in PBS without $\mathrm{Mg}^{2+} / \mathrm{Ca}^{2+}$ (Invitrogen, USA). Following this Neurobasal A medium supplemented with kanamycin (1\%) (Invitrogen, USA) was added to the cells. After $24 \mathrm{~h}$ the conditioned media (CM) was collected and frozen at $-80{ }^{\circ} \mathrm{C}$ until use.

\section{Primary Culture of Human Telencephalon Neural Progenitors (htNPCs) and Incubation with HUCPVCs-CM}

htNPCs were obtained from Prof. Leo A. Behie (University of Calgary, Calgary, Canada). Cells were isolated from the telencephalon region according with the protocols and strict ethical guidelines previously established [38-40]. htNPCs were thawed at $37{ }^{\circ} \mathrm{C}$ and the content placed in a T-25 flask (Nalge Nunc, Rochester, NY) containing $5 \mathrm{~mL}$ of a serum-free medium PPRFh2 [38]. After 2 days, the cells were harvested and mechanically dissociated into a single cell suspension, and subcultured into fresh cell growth medium (PPRF-h2). Every 4 days, the T-flaks were fed by replacing $40 \%$ of the spent medium with fresh growth medium. After 14-20 days of growth in the culture flasks, htNPCs were passaged and plated on a pre-coated (poly-D-lysine hydrobromide $(100 \mu \mathrm{g} / \mathrm{mL})$ and laminin $(10 \mu \mathrm{g} / \mathrm{mL})$ - Sigma, USA) 24 -well plate at a density of $1.0 \times$ $10^{5}$ per well during 5 days with the HUCPVCs-CM at $37{ }^{\circ} \mathrm{C}$, $5 \% \mathrm{CO}_{2}, 95 \%$ air and $90 \%$ relative humidity. Neurobasal A medium with $1 \%$ of kanamycin was used as control group.

\section{Stereotaxic Surgeries}

All experiments were conducted using 8 weeks old male Wistar rats (Charles River, Spain). Consent from the Portuguese national authority for animal experimentation, Direção Geral de Veterinária (ID: DGV9457), was obtained before the experiments. Animals were kept and handled in accordance with the guidelines for the care and handling of laboratory animals in the Directive 2010/63/EU of the European Parliament and of the Council. The animals were housed and maintained in a controlled environment at $22-24{ }^{\circ} \mathrm{C}$ and $55 \%$ humidity, on $12 \mathrm{~h}$ light/dark cycles and fed with regular rodent's chow and tap water ad libitum. Animals were handled for 1 week prior to the beginning of the injections, in order to reduce the stress induced by the surgical procedures. For the cerebral injections three experimental groups were used ( $n=5 /$ group): 1) Sham, 2) HUCPVCs and 3) HUCPVCs-CM. Adult rats were anesthetized with ketamine hydrochloride $(150 \mathrm{mg} / \mathrm{kg})$ plus medetomidine $(0.3 \mathrm{mg} / \mathrm{kg})$. Using a stereotaxic system (Stoelting, USA) and a Hamilton syringe ( $0.5 \mu \mathrm{l}$ Hamilton, Switzerland) all injections made in these three groups were bilateral according to previously determined coordinates (Anterior $/$ Posterior $(\mathrm{AP})=3.5 \mathrm{~mm}$;
Dorsal $/$ Ventral $(\mathrm{DV})=3.5 / 3.1 \mathrm{~mm}$; Lateral $(\mathrm{L})=2.0 \mathrm{~mm})[41]$. Coordinates had been previously confirmed by a preliminary surgery with methylene blue injected into the desired area (data not shown). The volume injected per DG was $0.5 \mu \mathrm{l}$ with a rate of injection of $0.25 \mu \mathrm{l}$ per minute. Two minutes were allowed after each injection in order to avoid any backflow up the needle tract. Sham group was only injected with $0.5 \mu$ of Neurobasal A medium; HUCPVCs group with $5.0 \times 10^{4}$ cells (in $0.5 \mu \mathrm{l}$ of Neurobasal A medium) and finally, the $\mathrm{CM}$ group were injected with $0.5 \mu \mathrm{l}$ of HUCPVCs-CM. At the end, the animals were sutured and then injected with $100 \mu \mathrm{l}$ of anti-sedating (Orion Pharma, Finland) in order to recover from surgical procedure.

\section{BrdU Administration and Histological Procedures}

Adult rats received intraperitoneally (i.p.) $100 \mathrm{mg} / \mathrm{kg}$ of BrdU (Sigma, USA) $24 \mathrm{~h}$ before the sacrifice. 7 days after of the stereotaxic injection animals were anaesthetized with sodium pentobarbital (Eutasil, $60 \mathrm{mg} / \mathrm{kg}$ i.p.; Ceva Saúde Animal, Portugal) and perfused with paraformaldehyde (4\%) (Merck, Lisbon, Portugal) diluted in PBS $(0,1 \mathrm{x})$ in order to fix the tissues. Subsequently, the brains were stored in sucrose solution (30\%) before heading to histological processing.

\section{Immunostaining}

htNPCs were fixed in $4 \%$ paraformaldehyde for $15 \mathrm{~min}$, and then permeabilized by incubation with $0.1 \%$ Triton X-100 in PBS for $5 \mathrm{~min}$ at room temperature, and washed three times in PBS. hNPCs were then blocked with $10 \%$ of fetal calf serum (FCS, Invitrogen, USA) in PBS, being followed by a $1 \mathrm{~h}$ incubation (at room temperature) with the primary antibodies: rabbit anti-doublecortin (DCX; 1:500, Abcam, USA) to detect immature neurons and mouse anti-rat microtubule associated protein-2 (MAP-2; 1:500, Sigma, USA) to detect mature neurons. htNPCs were then washed in PBS three times and incubated with the secondary antibodies namely, Alexa Fluor 488 goat anti-rabbit (IgG, Invitrogen, USA) and Alexa Fluor 594 goat anti-mouse immunoglobulin $\mathrm{G}$ (IgG; Invitrogen) during $1 \mathrm{~h}$ at room temperature. Finally a $10 \mathrm{~min}$ incubation with 4-6diamidino-2-phenylindole-dihydrochloride (DAPI; Invitrogen, USA) was performed. Samples were then observed under an Olympus BX-61 Fluorescence Microscope (Olympus, Germany). For this purpose, three coverslips and ten representative fields per condition were chosen and analyzed. Results are shown as percentage of DCX or MAP-2 positive cells over the total number of cells per field of observation $(n=3)$.

Regarding the in vivo experiment, coronal sections were obtained by vibrotome (VT1000S, Leica, Germany) with a thickness of $40 \mu \mathrm{m}$ and processed as free-floating sections. As first approach, sections were permeabilized in PBS-T (0.2 \%) (three times for $10 \mathrm{~min}$ ), and were subsequently incubated with $10 \%$ FCS/PBS during $2 \mathrm{~h}$ for endogenous blocking. After that, 
sections were incubated overnight at $4{ }^{\circ} \mathrm{C}$ with primary antibodies namely, DCX (1:300) for immature neurons, rabbit anti-rat glial fibrillary acid protein (GFAP; 1:200 Millipore, USA) for astrocytes detection; Human nuclear antigen (HNA; 1:100, Millipore, USA) for HUCPVCs detection; and 5bromo-2-deoxyuridine (BrdU; 1:100, Abcam, USA) for proliferation. Sections were then incubated with secondary antibodies: Alexa Fluor 568 goat antimouse immunoglobulin G (IgG; Invitrogen, USA), Alexa Fluor 488 goat anti-rabbit (IgG, Invitrogen, USA) during $2 \mathrm{~h}$ at room temperature, followed by a $10 \mathrm{~min}$ incubation with DAPI. Images were obtained with a confocal microscope (Olympus FV1000, Germany) using the software FV10-ASW 2.0c (Olympus, Germany), presenting the hippocampal DG (five sections per animal were analyzed for a $n=5 /$ group). Then, SGZ/GCL area was defined and the cell counts were made in these areas.

\section{Western Blot}

Rat hippocampi ( $n=4 /$ group) were homogenized in cold RIPA buffer (50 mM Tris-HCl, $150 \mathrm{mM} \mathrm{NaCl}, 0.1 \%$ SDS, $1 \% \mathrm{NP}-$ 40, PMSF and a protease inhibitors cocktail (Complete; Roche). Protein concentration was determined using the Bradford assay (BioRad). Samples were sonicated for $5 \mathrm{~min}$, boiled for $5 \mathrm{~min}$ at $100{ }^{\circ} \mathrm{C}$, and centrifuged for $10 \mathrm{~s}$ before loading. Fifty micrograms of total protein were loaded into $15 \%$ SDS-Page gels and then transferred to nitrocellulose membranes. After overnight incubation at $4{ }^{\circ} \mathrm{C}$ with the primary antibodies: rabbit anti-FGF2 (1:100, Abcam), rabbit anti-NGF (1:100, Abcam), the secondary antibodies were incubated $1 \mathrm{~h}$ at room temperature at the following dilutions: anti-rabbit (1:10.000, BioRad) and anti-mouse (1:15.000, BioRad). Antibody affinity was detected by chemiluminescence (ECL kit, BioRad). Band quantification was performed using ImageJ software according to the manufacturer's instructions using alpha-tubulin as the loading control.

\section{Statistical Analysis}

Statistical evaluation was performed using One-way ANOVA and Student's t-test through the program GraphPad Prism five
(GraphPad Software Inc., La Jolla, CA, USA). Data is presented as mean \pm standard deviation. Significance value was set at $p<0.05$.

\section{Results}

\section{Characterization of HUCPVCs}

The isolation of HUCPVCs from the perivascular region of the human umbilical cord has previously been reported [27]. In the present study, it was possible to observe HUCPVCs in culture, presenting the morphological characteristics typical of MSCs (Fig. 1a). The differentiation into mesodermal lineages is considered another basic characteristic of MSCs [42, 43]. We were also able to observe the multipotent character of HUCPVCs, obtained through the differentiation towards the osteogenic (Fig. 1b, formation of bone nodules) and adipogenic (Fig. 1c, formation of fat droplets) lineages. These results confirm that HUCPVCs are capable to differentiate into different lineages similarly to other MSC populations $[26,44,45]$.

HUCPVCs-CM Induced Neuronal Differentiation of hNPCs in vitro

htNPCs grow as neurospheres in a serum-free medium, PPRFh2 (Fig. 2a). However, when the growth medium is removed and replaced by the HUCPVCs-CM or Neurobasal A medium, htNPCs lose the neurosphere state and start to differentiate (Fig. 2b). Immunocytochemistry analysis revealed that when htNPCs were incubated for 5 days with the HUCPVCs-CM there was a clear increase $(p<0.01)$ of DCX positive (immature neurons; Fig. 3b/c) and MAP-2 positive cells (mature neurons; Fig. 3e/f) densities when compared to control group (incubation with Neurobasal A medium), as the latter had almost undetected numbers of DCX and MAP-2 positive cells (Fig. 3a/d).
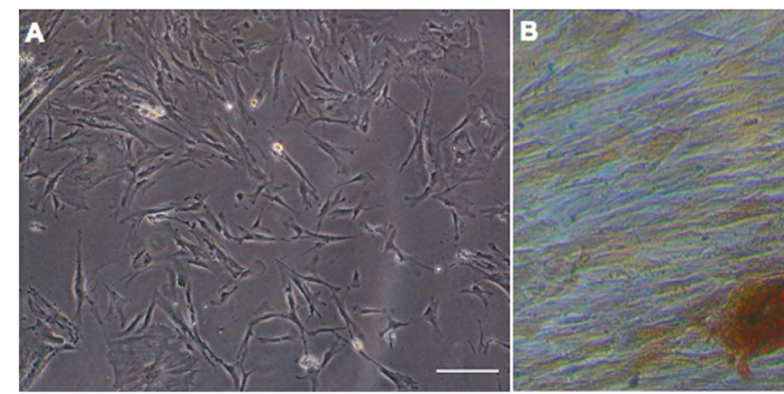

Fig. 1 Multipotent character of HUCPVCs in vitro. (a) HUCPVCs under standard culturing conditions, and differentiated into (b) osteogenic and (c) adipogenic lineages. Osteogenic differentiation was assessed using

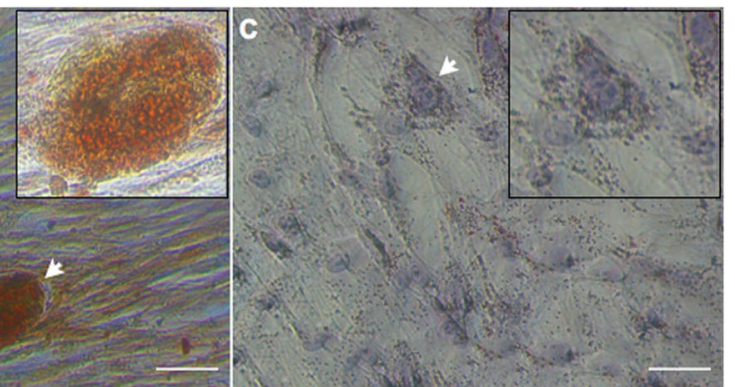

Alizarin Red staining, while adipogenic phenotype was assessed through the use of Oil Red O staining (Scale bar: (a) $100 \mu \mathrm{m},(\mathbf{b}, \mathbf{c}) 50 \mu \mathrm{m}$ ) 
Fig. 2 Expansion of Human Telencephalon Neural Precursor Cells (htNPCs) in vitro. a htNPCs standard expansion as neurospheres in the presence of their growth medium PPRF-h2 and b) spontaneous

differentiation into neural phenotypes upon PPRF-h2 removal. (Scale bar: $50 \mu \mathrm{m})$
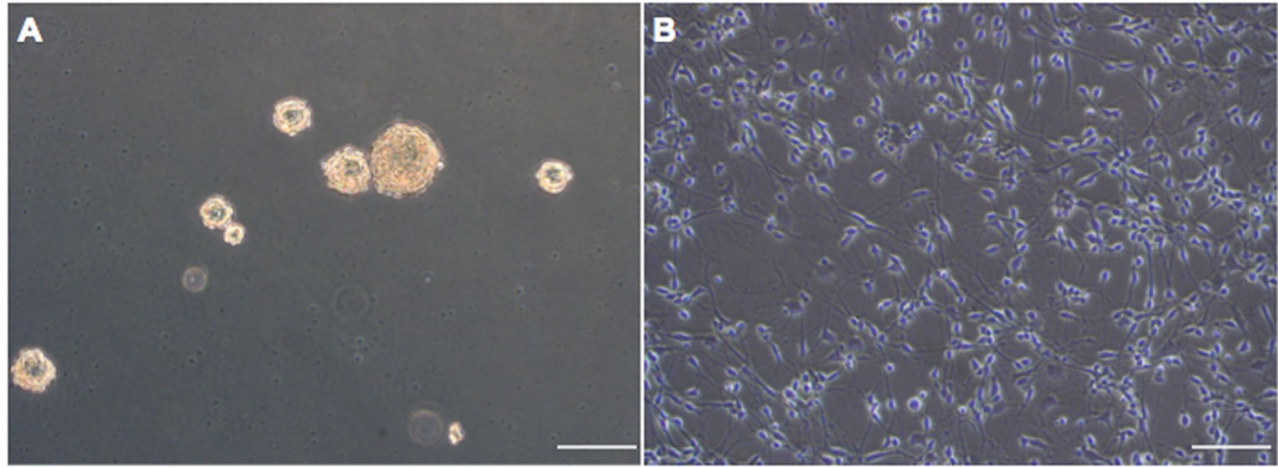

HUCPVCs and their CM Promoted Proliferation and Differentiation of Endogenous Progenitor Cells

To assess their role in vivo HUCPVCs (50.000 cells/ DG) and HUCPVCs-CM (0.5 $\mu \mathrm{L} / \mathrm{DG})$ were bilaterally injected into the hippocampal DG of adult rats without any concomitant immunosuppression therapy. As a first approach, we determined if HUCPVCs were able to survive into the DG 7 days after the transplantation. As it can be observed in Fig. 4a, the transplanted cells detected by immunostaining for human nuclear antigen (HNA), were able to survive in the DG 7 days after the transplantation.

In order to confirm proliferation and the cell type of the newly-born cells in the hippocampal DG, sections were immunostained with different markers. BrdUpositive cells were found mainly in the SGZ of the hippocampus. We first verified if the proliferating cells could be derived from HUCPVCs, but no positive colabeling between BrdU and HNA was observed (Fig. 4b). Nevertheless, the analysis of the cell proliferation in the DG, revealed a significant increase in the number of $\mathrm{BrdU}^{+}$cells within the HUCPVCs-injected DG $(p<0.05$; Fig. 4c) when compared to the control group (Sham, Fig. 4c). Similar findings were observed in the group injected with HUCPVCs-CM, $(p<0.05$, Fig. 4c).

After observing that HUCPVCs and their respective CM were able to stimulate the proliferation in the DG, we next aimed to determine their effects on the differentiation of $\mathrm{DG}$
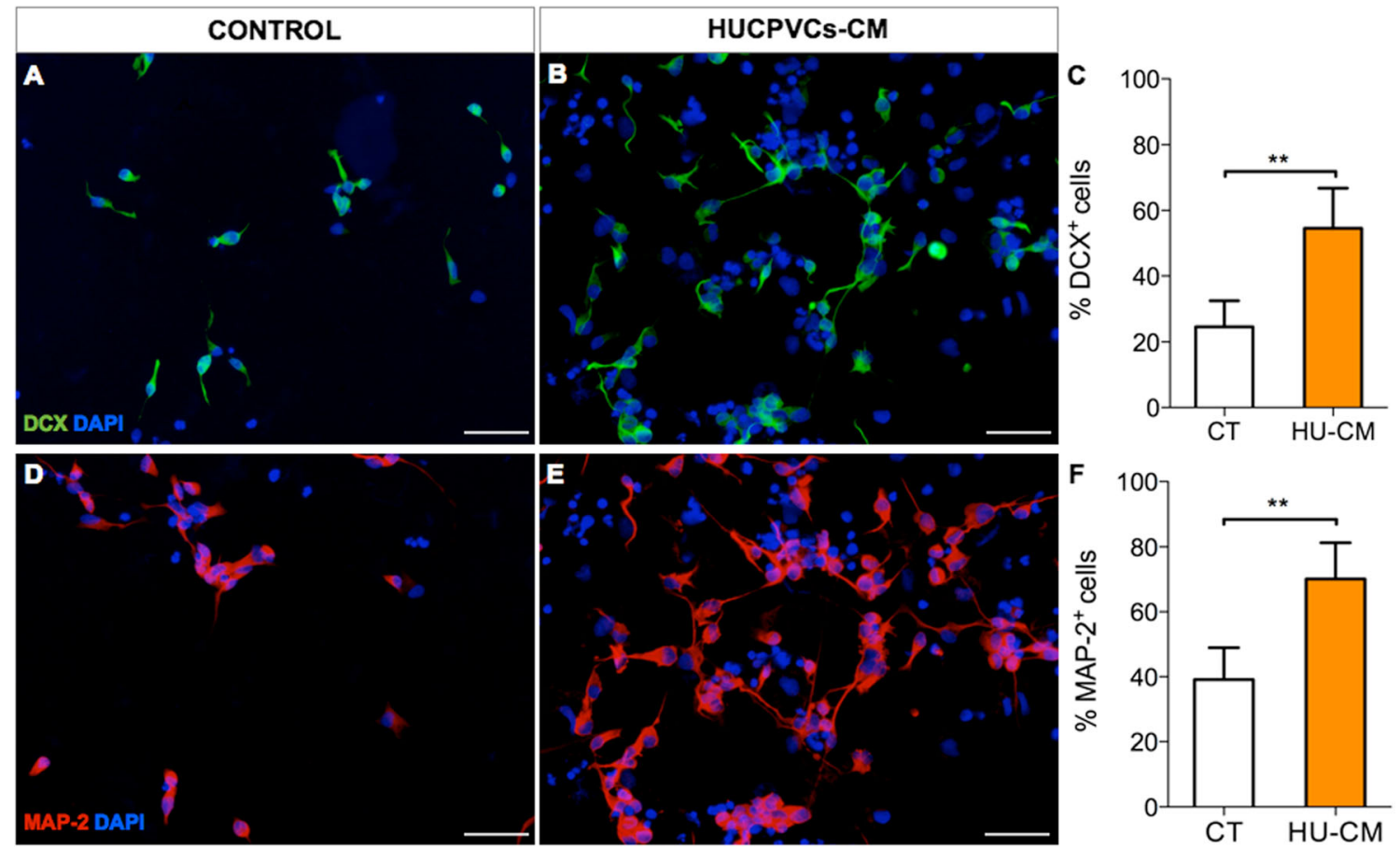

Fig. 3 In vitro neuronal differentiation of htNPCs. HUCPVCs-CM was able to significantly increase the survival and differentiation of htNPCs into neuronal phenotypes such as (b) immature $\left(\mathrm{DCX}^{+}\right.$cells) and (e) mature (MAP- $2^{+}$cells) neurons when compared to control conditions $(\mathbf{a} / \mathbf{d})(\mathbf{c} / \mathbf{f}$, mean $\pm \mathrm{SD}, n=3, p<0.05)$. CT Control (Neurobasal A media), $H U$-CM (HUCPVCs conditioned media). (Scale bar: $50 \mu \mathrm{m}$ ) 

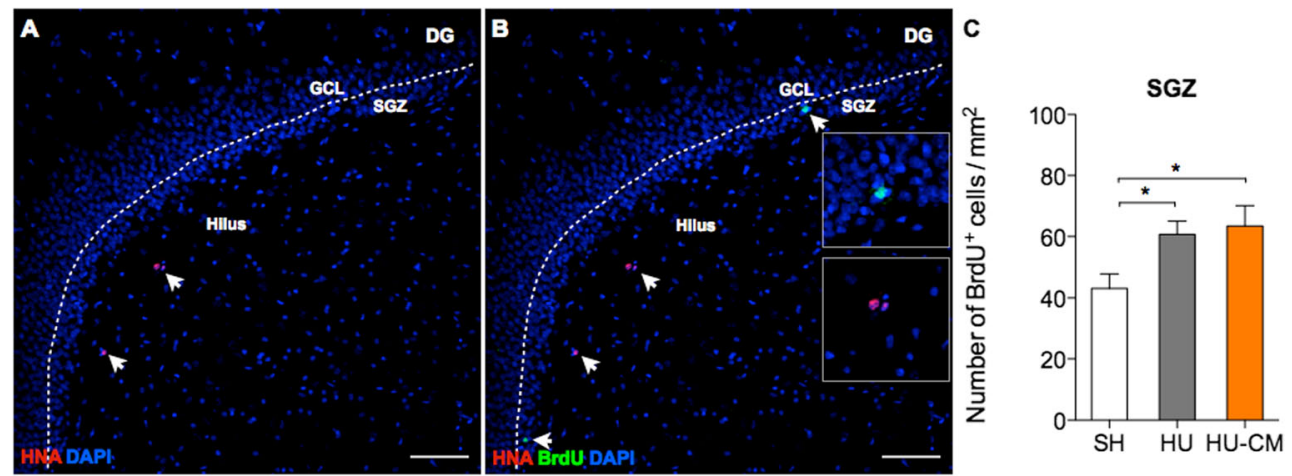

Fig. 4 Transplantation of HUCPVCs and their CM into the hippocampal DG. HUCPVCs were able to survive in vivo 7 days after transplantation $\left(\mathrm{HNA}^{+}\right.$cells, red) (a). However, they were not proliferating, as there was no co-localization between $\mathrm{HNA}^{+}$and $\mathrm{BrdU}^{+}$cells. Cell counts (c) revealed that 7 days post-injection both HUCPVCs and CM transplanted

groups were able to increase the number of BrdU-labeled cells in the DG, when compared to control (Sham group; mean $\pm \mathrm{SD}, n=5, p<0.05$ ). $S H$ (Sham), HU (HUCPVCs), HU-CM (HUCPVCs conditioned media). (Scale bar: $100 \mu \mathrm{m}$ )

resident cells. 7 days post-injection it was observed that both HUCPVCs (Fig. 5b) and their CM (Fig. 5c) were able to increase $(p<0.05)$ the number of DCXexpressing cells in the SGZ (Fig. 5d) when compared to the Sham group (Figs. 5a); for the GCL, differences were only observed between HUCPVCs-CM and sham groups, respectively (Fig. 5e, $p<0.05$ ). Regarding the effects on the astrocytic cell densities (Fig. 6a-c), differences were only observed $(p<0.05)$ for the HUCPVCs-CM group in the SGZ (Fig. 6d). No differences were observed in the GCL.
Expression of Trophic Factors

after HUCPVCs/HUCPVCs-CM Transplantation or Injection

In order to stimulate the endogenous proliferation as well as to increase the number of neuronal/glial cell populations, HUCPVCs or their CM must be able to modulate the surrounding environment through direct interaction or paracrine action [45]. Therefore the levels of FGF-2 and NGF in the DG were determined. Western blot analysis (Fig. 7) revealed that levels of FGF-2 were significantly increased when compared to those obtained by the Sham group ( $p<0.05$; Figs. $7 \mathrm{a} / \mathrm{b}$ ).
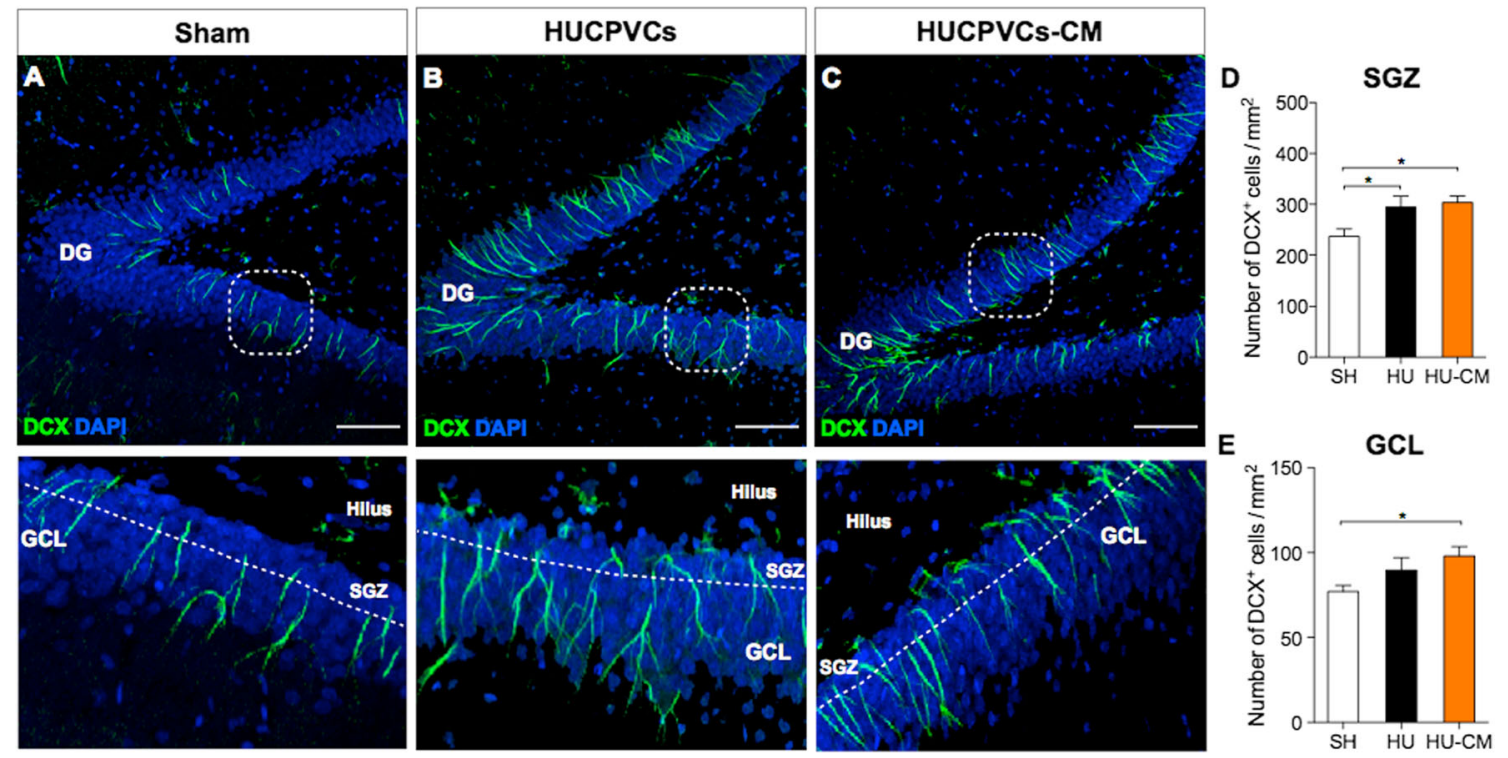

Fig. 5 HUCPVCs-CM enhances neuronal densities in the DG of hippocampus. Immunohistochemistry for DCX (newborn neurons: $\mathbf{a} / \mathbf{b}$ / c) revealed that the CM of HUCPVCs was able to increase the number of newborn neurons (c, statistically significant to the Sham group; mean \pm $\mathrm{SD}, n=5, p<0.05$ ) both in the (d) SGZ and (e) GCL. HUCPVCs were

also able to increase the number of immature neurons (B, statistically significant to the Sham group; mean $\pm \mathrm{SD}, n=5, p<0.05)$ into the (d) SGZ. SH (Sham), HU (HUCPVCs), HU-CM (HUCPVCs conditioned media). (Scale bar: $100 \mu \mathrm{m})$ 

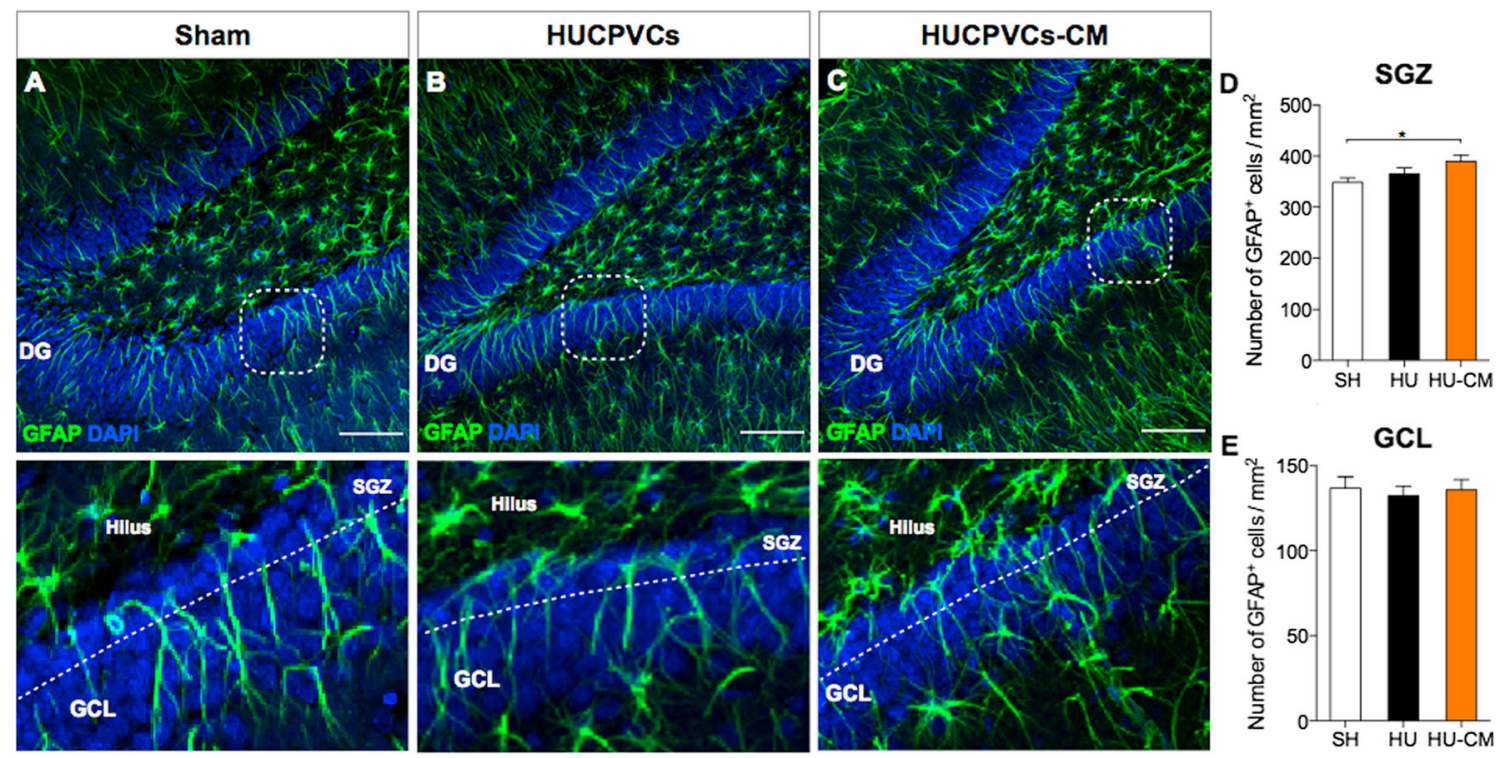

Fig. 6 HUCPVCs-CM enhances astrocytic densities in the DG of hippocampus. Immunohistochemistry to GFAP (Astrocytes: a/b/c) revealed increased numbers promoted by the injection of HUCPVCs$\mathrm{CM}$ (c, statistically significant to the Sham group; mean $\pm \mathrm{SD}, n=5$,

\section{Discussion}

The use of MSCs-based therapies as a new strategy for CNS regeneration constitutes one of the biggest challenges for regenerative medicine. Different reports have shown that the release of paracrine factors and extracellular vesicles is the main route by which MSCs can mediate improvements in the CNS [34]. Within the different MSCs based populations, HUCPVCs have been described as an attractive and readily available alternative source of MSCs for cell therapy [27]. Indeed, studies have shown that HUCPVCs have a higher clonogenic frequency and display a robust self-renewing capacity. Additionally, they have also shown to possess multilineage differentiation [46, 47], as confirmed in the present report (Fig. 1). Moreover, previous results from our laboratory revealed that the secretome of these cells was able to $p<0.05)$ in the (d) SGZ. In the (e) GCL no differences were observed 7 days post-injection. $S H$ (Sham), $H U$ (HUCPVCs), $H U-C M$ (HUCPVCs conditioned media) (Scale bar: $100 \mu \mathrm{m})$

induce and increase the cell survival and densities of different (hippocampal, cortical and cerebellar) neuronal and glial cells $[23,33,37,48]$. The initial in vitro experiments performed in the present report revealed that the secretome of HUCPVCs could also modulate the differentiation of CNS derived cells. Indeed, as shown in Fig. 3 when htNPCs were incubated with HUCPVCs-CM an increased differentiation of htNPCs into neuronal lineages (immature $\left(\mathrm{DCX}^{+}\right)$and mature (MAP- $\left.2^{+}\right)$ neurons) was observed. Previous work from Ribeiro et al. [33] had already shown that these cells have a strong expression of NGF, which is most likely playing a strong role on the observed effects.

In vivo experiments revealed that HUCPVCs were able to boost cell proliferation within the DG 7 days after injection, as assessed by the number of $\mathrm{BrdU}^{+}$cells (Fig. 4c). Similar outcomes were reported by Munoz and colleagues [49] after
A

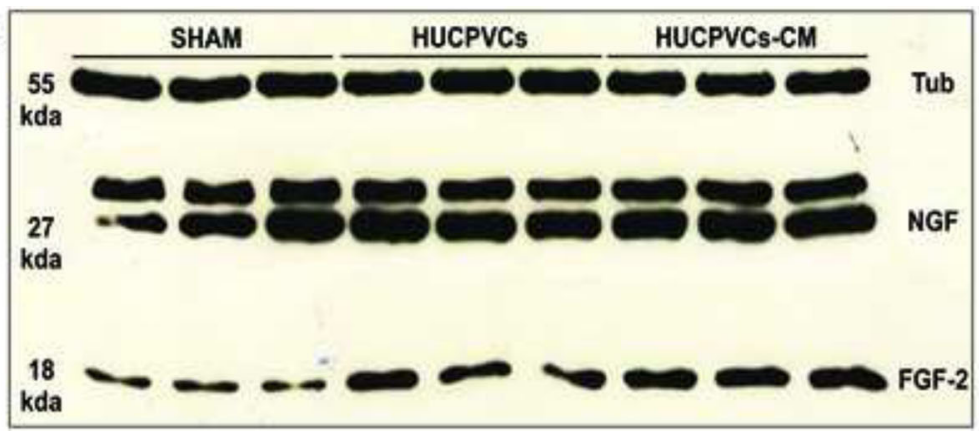

B

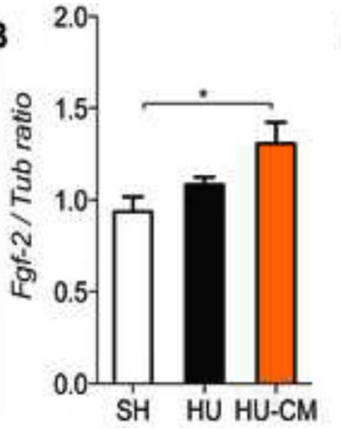

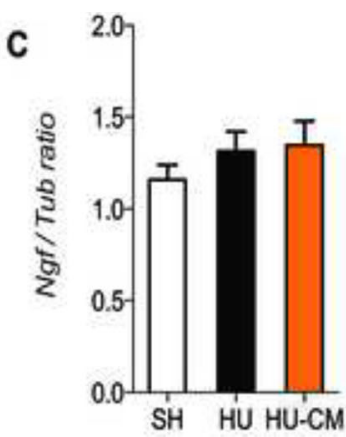

Fig. 7 Injection of HUCPVCs-CM increased the expression of neurotrophic factors in vivo. By Western blot it was possible to observe that HUCPVCs-CM was able to increase the secretion of (a) FGF-2 in the DG tissue (b, statistically significant to the Sham group; mean $\pm \mathrm{SD}, n=4$, $p<0.05$ ). It was also observed a slightly increase in the levels of (c) NGF, although no statistically significant differences were observed when compared to the Sham group. SH (Sham), HU (HUCPVCs), HU-CM (HUCPVCs conditioned media) 
BM-MSCs transplantation in the DG, being this finding attributed to the secretion of growth factors and chemokines; this hypothesis was, in fact, confirmed in the present study. Indeed, as shown in Fig. 4c, the application of CM discloses similar levels of cell proliferation to those obtained by the cell transplanted group. This is a strong indicator that the secretome, per se, regulates such phenomena.

Similar effects were also observed regarding neuronal differentiation. Indeed, 7 days after injection of HUCPVCs, or their respective $\mathrm{CM}$, it was possible to observe a significantly increase number of immature neurons $\left(\mathrm{DCX}^{+}\right.$cells) in the SGZ (Fig. 5d). This is in line with the findings of Tfilin and colleagues [50], who transplanted BM-MSCs into the hippocampal DG, and observed that after the transplantation there were increasing numbers of DCX-expressing cells, indicating the differentiation of newly formed neurons. Curiously, in the present study it was observed, that the CM, and not the cells, was also able to increase significantly the number of DCXexpressing cells into the GCL (Fig. 5e), suggesting that the secretome could stimulate the migration of these newly-born neurons within the DG. We hypothesized that these effects are related with the increased expression of FGF-2 in the CM injected group (Fig. 7a/b). Indeed, the latter is considered to be a potent modulator of the early phases of neurogenesis, being able to support not only the proliferation as well as, to enhance the survival and neuronal/glial maturation $[45,50]$. The small increase of NGF in the DG tissue (Fig. 7a/c) could also impact on these phenomena [49, 51-53]. Nevertheless, although proteomic studies are still scarce to explain the functional effects of MSCs secretome (including that of HUCPVCs as well), previous studies from our group have revealed, through the use of targeted and non-targeted proteomic-based techniques, the presence of important molecules for CNS physiology, within it [33, 37]. For instance, Ribeiro and colleagues [33] demonstrated that HUCPVCs were able to robustly secrete $\mathrm{NGF}$, an important neurotrophin for the support of neuronal/ glial cell survival $[51,52]$. In a subsequent study, it was also possible to observe that HUCPVCs were also able to secrete important intracellular proteins such as 14-3-3, Hsp70 and UCHL1, which are known for their important roles in the induction of cell proliferation, survival and differentiation, including in cells derived from the CNS [54-56]. Therefore it is possible that these molecules are playing a role on the phenomena exerted in vivo by HUCPVCs secretome.

\section{Conclusions}

In the present work it was demonstrated that the secretome of HUCPVCs, a MSC based population within the Wharton Jelly of the umbilical cord, was able to induce neuronal differentiation of human neural progenitors into neurons in different stages of maturation. Additionally, in vivo injections into the hippocampal DG, a known neurogenic niche, revealed that both HUCPVCs or CM injected groups, had increased levels of $\mathrm{BrdU}^{+}$cells (proliferation) and newly differentiated neurons $\left(\mathrm{DCX}^{+}\right)$, when compared to sham animals. These outcomes can be associated with the expression of FGF-2 and other proteins, which have been previously shown to constitute part of the secretome of these cells. These results suggest that the secretome (alone) of a MSC-like population is able to modulate neural progenitor proliferation and differentiation, which may open new therapeutic opportunities in the future.

Acknowledgments Foundation Calouste Gulbenkian for funds under the scope of the Gulbenkian Programme to Support Cutting Edge Research in Life Sciences; Portuguese Foundation for Science and Technology (FCT) for Ciência 2007 program and IF Development Grant (A.J. Salgado), and pre-doctoral fellowship to F.G. Teixeira (SFRH / BD / 69637 / 2010); John E. Davies for kindly providing the HUCPVCs used in this work.

Conflict of Interest The author(s) declare that they have no competing interests.

\section{References}

1. Balu, D. T., \& Lucki, I. (2009). Adult hippocampal neurogenesis: regulation, functional implications, and contribution to disease pathology. Neuroscience and Biobehavioral Reviews, 33(3), 232-52.

2. Kazanis, I. (2009). The subependymal zone neurogenic niche: a beating heart in the centre of the brain: how plastic is adult neurogenesis? Opportunities for therapy and questions to be addressed. Brain, 132(Pt 11), 2909-21.

3. Kazanis, I., Belhad, I. A., Faissner, A., \& Ffrench-Constant, C. (2007). The adult mouse subependymal zone regenerates efficiently in the absence of tenascin-C. Journal of Neuroscience, 27(51), 13991-6.

4. Kan, I., Barhum, Y., Melamed, E., \& Offen, D. (2011). Mesenchymal stem cells stimulate endogenous neurogenesis in the subventricular zone of adult mice. Stem Cell Reviews, 7(2), 404-12.

5. Lindvall, O., \& Kokaia, Z. (2010). Stem cells in human neurodegenerative disorders-time for clinical translation? Journal of Clinical Investigation, 120(1), 29-40.

6. Shihabuddin, L. S., \& Aubert, I. (2010). Stem cell transplantation for neurometabolic and neurodegenerative diseases. Neuropharmacology, 58(6), 845-54.

7. Pittenger, M. F., Mackay, A. M., Beck, S. C., Jaiswai, R. K., Douglas, R., Mosca, J. D., et al. (1999). Multilineage potential of adult human mesenchymal stem cells. Science, 284(5411), 143-7.

8. Zuk, P. A., Zhu, M., Ashjian, P., De Ugarte, D. A., Huang, J. I., Mizuno, H., et al. (2002). Human adipose tissue is a source of multipotent stem cells. Molecular Biology of the Cell, 13(12), 4279-95.

9. Wang, H. S., Hung, S. C., Peng, S. T., Huang, C. C., Wei, H. M., Guo, Y. J., et al. (2004). Mesenchymal stem cells in the Wharton's jelly of the human umbilical cord. Stem Cells, 22(7), 1330-7.

10. Daher, S. R., Johnstone, B. H., Phinney, D. G., \& March, K. L. (2004). Adipose stromal/stem cells: basic and translational advances: the IFATS collection. Stem Cells, 26(10), 2664-5. 
11. Phinney, D. G., \& Isakova, I. (2005). Plasticity and therapeutic potential of mesenchymal stem cells in the nervous system. Current Pharmaceutical Design, 11(10), 1255-65.

12. Prockop, D. J., Azizi, S. A., Colter, D., Digirolamo, C., Kopen, G., \& Phinney, D. G. (2000). Potential use of stem cells from bone marrow to repair the extracellular matrix and the central nervous system. Biochemical Society Transactions, 28(4), 341-5.

13. Wei, X., Zhao, L., Zhong, J., Gu, H., Feng, D., Johnstone, B. H., et al. (2009). Adipose stromal cells-secreted neuroprotective media against neuronal apoptosis. Neuroscience Letters, 462(1), 76-9.

14. Honmou, O., Onodera, R., Sasaki, M., Waxman, S. G., \& Kocsis, J. D. (2012). Mesenchymal stem cells: therapeutic outlook for stroke. Trends in Molecular Medicine, 18(5), 292-7.

15. Constantin, G., Marconi, S., Rossi, B., Angiari, S., Anghileri, E., Gini, B., et al. (2009). Adipose-derived mesenchymal stem cells ameliorate chronic experimental autoimmune encephalomyelitis. Stem Cells, 27(10), 2624-35.

16. Cristofanilli, M., Harris, V. K., Zigelbaum, A., Goossens, A. M., Lu, A., Rosenthal, H., et al. (2011). Mesenchymal stem cells enhance the engraftment and myelinating ability of allogeneic oligodendrocyte progenitors in dysmyelinated mice. Stem Cells and Development, 20(12), 2065-76.

17. Cova, L., Armentero, M. T., Zennaro, E., Calzarossa, C., Bossolasco, P., Busca, G., et al. (2010). Multiple neurogenic and neurorescue effects of human mesenchymal stem cell after transplantation in an experimental model of Parkinson's disease. Brain Research, 1311, $12-27$.

18. Erba, P., Terenghi, G., \& Kingham, P. J. (2010). Neural differentiation and therapeutic potential of adipose tissue derived stem cells. Current Stem Cell Research \& Therapy, 5(2), 153-60.

19. Arboleda, D., Forostyak, S., Jendelova, P., Marekova, D., Amemori, T., Pivonkova, H., et al. (2011). Transplantation of predifferentiated adipose-derived stromal cells for the treatment of spinal cord injury. Cellular and Molecular Neurobiology, 31(7), 1113-22.

20. Park, J. H., Kim, D. Y., Sung, I. Y., Choi, G. H., Jeon, M. H., Kim, K. K., et al. (2012). Long-term results of spinal cord injury therapy using mesenchymal stem cells derived from bone marrow in humans. Neurosurgery, 70(5), 1238-47. discussion 1247.

21. Taghizadeh, R. R., Cetrulo, K. J., \& Cetrulo, C. L. (2011). Wharton's Jelly stem cells: future clinical applications. Placenta, 32(Suppl 4), S311-5.

22. Datta, I., Mishra, S., Mohanty, L., Pulikkot, S., \& Joshi, P. G. (2011). Neuronal plasticity of human Wharton's jelly mesenchymal stromal cells to the dopaminergic cell type compared with human bone marrow mesenchymal stromal cells. Cytotherapy, 13(8), 918-32.

23. Salgado, A. J., Fraga, J. S., Mesquita, A. R., Neves, N. M., Reis, R. L., \& Sousa, N. (2010). Role of human umbilical cord mesenchymal progenitors conditioned media in neuronal/glial cell densities, viability, and proliferation. Stem Cells and Development, 19(7), 1067-74.

24. Weiss, M. L., \& Troyer, D. L. (2006). Stem cells in the umbilical cord. Stem Cell Reviews, 2(2), 155-62.

25. Sarugaser, R., Ennis, J., Stanford, W. L., \& Davies, J. E. (2009). Isolation, propagation, and characterization of human umbilical cord perivascular cells (HUCPVCs). Methods in Molecular Biology, 482, 269-79.

26. Baksh, D., Yao, R., \& Tuan, R. S. (2007). Comparison of proliferative and multilineage differentiation potential of human mesenchymal stem cells derived from umbilical cord and bone marrow. Stem Cells, 25(6), 1384-92.

27. Sarugaser, R., Lickorish, D., Baksh, D., Hosseini, M. M., \& Davies, J. E. (2005). Human umbilical cord perivascular (HUCPV) cells: a source of mesenchymal progenitors. Stem Cells, 23(2), 220-9.

28. Weiss, M. L., Medicetty, S., Bledsoe, A. R., Rachakatia, R. S., Choi, M., Merchav, S., et al. (2006). Human umbilical cord matrix stem cells: preliminary characterization and effect of transplantation in a rodent model of Parkinson's disease. Stem Cells, 24(3), 781-92.
29. Hirko, A. C., Dallasen, R., Jomura, S., \& Xu, Y. (2008). Modulation of inflammatory responses after global ischemia by transplanted umbilical cord matrix stem cells. Stem Cells, 26(11), 2893-901.

30. Yang, C. C., Shih, Y. H., Ko, M. H., Hsu, S. Y., Cheng, H., \& Fu, Y. S. (2008). Transplantation of human umbilical mesenchymal stem cells from Wharton's jelly after complete transection of the rat spinal cord. PloS One, 3(10), e3336.

31. Zhang, L., Zhang, H. T., Hong, S. Q., Ma, X., Jiang, X. D., \& Xu, R. X. (2009). Cografted Wharton's jelly cells-derived neurospheres and BDNF promote functional recovery after rat spinal cord transection. Neurochemical Research, 34(11), 2030-9.

32. Carvalho, M. M., Teixeira, F. G., Reis, R. L., Sousa, N., \& Salgado, A. J. (2011). Mesenchymal stem cells in the umbilical cord: phenotypic characterization, secretome and applications in central nervous system regenerative medicine. Current Stem Cell Research \& Therapy, 6(3), 221-8.

33. Ribeiro, C. A., Fraga, J. S., Graos, M., Neves, N. M., Reis, R. L., Gimble, J. M., et al. (2012). The secretome of stem cells isolated from the adipose tissue and Wharton jelly acts differently on central nervous system derived cell populations. Stem Cell Research \& Therapy, 3(3), 18.

34. Teixeira, F. G., Carvalho, M. M., Sousa, N., \& Salgado, A. J. (2013). Mesenchymal stem cells secretome: a new paradigm for central nervous system regeneration? Cellular and Molecular Life Sciences, 70(20), 3871-82.

35. Koh, S. H., Kim, K. S., Choi, M. R., Jung, K. H., Park, K. S., Chai, Y. G., et al. (2008). Implantation of human umbilical cord-derived mesenchymal stem cells as a neuroprotective therapy for ischemic stroke in rats. Brain Research, 1229, 233-48.

36. Ding, D. C., Shyu, W. C., Chiang, M. F., Lin, S. Z., Chang, Y. C., Wang, H. J., et al. (2007). Enhancement of neuroplasticity through upregulation of beta1-integrin in human umbilical cord-derived stromal cell implanted stroke model. Neurobiology of Disease, 27(3), 339-53.

37. Fraga, J. S., Silva, N. A., Lourenço, A. S., Gonçalves, V., Neves, N. M., Reis, R. L., et al. (2013). Unveiling the effects of the secretome of mesenchymal progenitors from the umbilical cord in different neuronal cell populations. Biochimie, 95(12), 2297-303.

38. Baghbaderani, B. A., Mukhida, K., Sen, A., Kallos, M. S., Hong, M., Mendez, I., et al. (2010). Bioreactor expansion of human neural precursor cells in serum-free media retains neurogenic potential. Biotechnology and Bioengineering, 105(4), 823-33.

39. Mendez, I., Dagher, A., Hong, M., Gaudet, P., Weerasinghe, S., McAlister, V., et al. (2002). Simultaneous intrastriatal and intranigral fetal dopaminergic grafts in patients with Parkinson disease: a pilot study. Report of three cases. Journal of Neurosurgery, 96(3), 589-96.

40. Mendez, I., Sanchez-Pernaute, R., Cooper, O., Vinuela, A., Ferrari, D., Bjorklund, L., et al. (2005). Cell type analysis of functional fetal dopamine cell suspension transplants in the striatum and substantia nigra of patients with Parkinson's disease. Brain, 128(Pt 7), 1498-510.

41. Paxinos, G., \& Watson, C. (2004). Rat brain in stereotaxic coordinates (5th ed.). San Diego: Academic.

42. Bernardo, M. E., Locatelli, F., \& Fibbe, W. E. (2009). Mesenchymal stromal cells. Annals of the New York Academy of Sciences, 1176, $101-17$.

43. Chen, Y., Shao, J. Z., Xiang, L. X., Dong, X. J., \& Zhang, G. R. (2008). Mesenchymal stem cells: a promising candidate in regenerative medicine. International Journal of Biochemistry and Cell Biology, 40(5), 815-20.

44. Baer, P.C. and Geiger, H. (2012). Adipose-derived mesenchymal stromal/stem cells: tissue localization, characterization, and heterogeneity. Stem Cells International, p. 812693.

45. Huang, A. H., Snyder, B. R., Cheng, P. H., \& Chan, A. W. (2008). Putative dental pulp-derived stem/stromal cells promote proliferation and differentiation of endogenous neural cells in the hippocampus of mice. Stem Cells, 26(10), 2654-63. 
46. Sarugaser, R., Hanoun, L., Keating, A., Stanford, W. L., \& Davies, E. (2009). Human mesenchymal stem cells self-renew and differentiate according to a deterministic hierarchy. PloS One, 4(8), e6498.

47. Yannarelli, G., Pacienza, N., Cuniberti, L., Medin, J., Davies, J., \& Keating, A. (2013). Brief report: the potential role of epigenetics on multipotent cell differentiation capacity of mesenchymal stromal cells. Stem Cells, 31(1), 215-20.

48. Ribeiro, C. A., Salgado, A. J., Fraga, J. S., Silva, N. A., Reis, R. L., \& Sousa, N. (2011). The secretome of bone marrow mesenchymal stem cells-conditioned media varies with time and drives a distinct effect on mature neurons and glial cells (primary cultures). Journal of Tissue Engineering and Regenerative Medicine, 5(8), 668-72.

49. Munoz, J. R., Stoutenger, B. R., Robinson, A. P., Spees, J. L., \& Prockop, D. J. (2005). Human stem/progenitor cells from bone marrow promote neurogenesis of endogenous neural stem cells in the hippocampus of mice. Proceedings of the National Academy of Sciences of the United States of America, 102(50), 18171-6.

50. Tfilin, M., Sudai, E., Merenlender, A., Gispan, I., Yadid, G., \& Turgeman, G. (2010). Mesenchymal stem cells increase hippocampal neurogenesis and counteract depressive-like behavior. Molecular Psychiatry, 15(12), 1164-75.

51. Frielingsdorf, H., Simpson, D., Thal, L. J., \& Pizzo, D. P. (2007). Nerve growth factor promotes survival of new neurons in the adult hippocampus. Neurobiology of Disease, 26(1), 47-55.
52. Manni, L., Rocco, M. L., Bianchi, P., Soligo, M., Guaragna, M., Barbar, S. P., et al. (2013). Nerve growth factor: basic studies and possible therapeutic applications. Growth Factors, $31(4), 115-22$.

53. Kurata, S., Goto, T., Gunjigake, K. K., Kataoka, S., Kuroishi, N. K., Ono, K., et al. (2013). Nerve growth factor involves mutual interaction between neurons and satellite glial cells in the rat trigeminal ganglion. Acta Histochem Cytochem, 46(2), 65-73.

54. Chen, J., Lee, C. T., Errico, S. L., Becker, K. G., \& Freed, W. J. (2007). Increases in expression of 14-3-3 eta and 14-3-3 zeta transcripts during neuroprotection induced by delta9tetrahydrocannabinol in AF5 cells. Journal of Neuroscience Research, 85(8), 1724-33.

55. Bonner, H. P., Concannon, C. G., Bonner, C., Woods, I., Ward, M. W., \& Prehn, J. H. (2010). Differential expression patterns of Puma and Hsp70 following proteasomal stress in the hippocampus are key determinants of neuronal vulnerability. Journal of Neurochemistry, 114(2), 606-16.

56. Sakurai, M., Ayukawa, K., Setsuie, R., Nishikawa, K., Hara, Y., Ohashi, H., et al. (2006). Ubiquitin C-terminal hydrolase L1 regulates the morphology of neural progenitor cells and modulates their differentiation. Journal of Cell Science, 119(Pt 1), 162-71. 